\title{
ON THE EXISTENCE OF PROPER NEARLY KENMOTSU MANIFOLDS
}

\author{
I. KÜPELI ERKEN, PIOTR DACKO, AND C. MURATHAN
}

\begin{abstract}
This is an expository paper, which provides a first approach to nearly Kenmotsu manifolds. The purpose of this paper is to focus on nearly Kenmotsu manifolds and get some new results from it. We prove that for a nearly Kenmotsu manifold is locally isometric to warped product of real line and nearly Kähler manifold. Finally, we prove that there exist no nearly Kenmotsu hypersurface $M^{2 n+1}$ of nearly Kähler manifold $N^{2 n+2}$. It is shown that a normal nearly Kenmotsu manifold is Kenmotsu manifold.
\end{abstract}

\section{Introduction}

Nearly Kaehler manifolds were defined by Gray [7. He carry on study of Nearly Kaehler manifolds 8, 9. Nearly Sasakian manifolds were introduced by Blair, Showers and Yano in 2]. Afterwards, Olszak studied nearly Sasakian manifolds in [11]. He gave properties of 5-dimensional nearly Sasakian non-Sasakian manifolds. Parallel to the study of [11, Endo studied nearly cosymplectic manifolds [6]. Recently, Cappelletti Montano and Dileo study Nearly Sasakian Geometry [4. While much of the similarity between nearly Sasakian manifolds and nearly cosymplectic manifolds are emphasized, and properties of these manifolds are investigated, nearly Kenmotsu manifolds are ignored. The notion of nearly Kenmotsu manifold was introduced in [14. In the present paper, we want to fill this gap in the study of nearly Kenmotsu manifolds. In literature we did not fall in with proper nearly Kenmotsu manifold examples. So one can ask the following question. Do there exist proper nearly Kenmotsu manifolds? In this paper we give a positive answer to the question for dimension $>5$. In this study we gave certain properties of such manifolds. Our work is structured as follows: In Section 2, we report some basic information about nearly Kenmotsu manifolds. In the next section, we give some curvature identies about nearly Kenmotsu manifolds and we prove that for a nearly Kenmotsu manifold, $H=0$ and the distribution $D$ is completely integrable. In the last section, we show that a normal nearly Kenmotsu manifold is Kenmotsu manifold and there exist no nearly Kenmotsu hypersurface $M^{2 n+1}$ of nearly Kaehler manifold $N^{2 n+2}$.

\section{Preliminaries}

In this paper all objects are to be considered as $C^{\infty}$-class, manifolds are assumed to be connected. We accept the following convention that $X, Y, Z, W \ldots$, will denote vector fields, if it is not otherwise stated.

Let $M$ be a $(2 n+1)$-dimensional differentiable manifold and $\phi$ is a $(1,1)$ tensor field, $\xi$ is a vector field, $\eta$ is a one-form, $g$ Riemannina metric on $M$. Then $(\phi, \xi, \eta, g)$ is called

2010 Mathematics Subject Classification. 53C25, 53C55, 53D15.

Key words and phrases. Almost Contact Metric Manifold, Kenmotsu Manifold, Nearly Kenmotsu Manifold. 
an almost contact metric structure on $M$, if

$$
\phi^{2}=-I d+\eta \otimes \xi, \quad \eta(\xi)=1, \quad g(\phi X, \phi Y)=g(X, Y)-\eta(X) \eta(Y) .
$$

and $M$ is said to be an almost contact metric manifold if it is endowed with an almost contact metric structure [1, 16. For such manifold

$$
\begin{gathered}
\eta(X)=g(X, \xi), \quad \phi(\xi)=0, \quad \eta \circ \phi=0, \\
g(X, \phi Y)+g(Y, \phi X)=0,
\end{gathered}
$$

tensor field $\Phi(X, Y)=g(X, \phi Y)$, is customary called fundamental form. In this paper we will refer to $\xi$, as Reeb vector field and $\eta$, as Reeb form. By $[\phi, \phi]$ we denote Nijenhuis torsion tensor of $\phi$, by definition

$$
[\phi, \phi](X, Y)=\phi^{2}[X, Y]+[\phi X, \phi Y]-\phi[X, \phi, Y]-\phi[X, \phi Y]
$$

where $[X, Y]$ denotes the Lie bracket of vector fields.

An almost contact metric manifold $(M, \phi, \xi, \eta)$ is called nearly Kenmotsu manifold [14], if

$$
\left(\nabla_{X} \phi\right) Y+\left(\nabla_{Y} \phi\right) X=-\eta(Y) \phi X-\eta(X) \phi Y
$$

where $\nabla$ is the Levi-Civita connection of $g$. Moreover, if $M$ satisfies

$$
\left(\nabla_{X} \phi\right) Y=g(\phi X, Y) \xi-\eta(Y) \phi X
$$

then it is called Kenmotsu manifold [10]. Every Kenmotsu manifold is a nearly Kenmotsu manifold but the converse is not true, which in fact will be proved in this paper. If $M$ is nearly Kenmotsu but non Kenmotsu we will call manifold is proper nearly Kenmotsu manifold.

Let $M$ be nearly Kenmotsu manifold. We define $(1,1)$-tensor field $H$, by $d \eta(X, Y)=$ $g(H X, Y)$. Later on we will show that $H=0$.

Proposition 1. For a nearly Kenmotsu manifold we have

$$
\begin{aligned}
& g\left(\nabla_{X} \xi, Y\right)+g\left(X, \nabla_{Y} \xi\right)=2 g(\phi X, \phi Y), \quad \nabla_{X} \xi=-\phi^{2} X+H X, \\
& \nabla_{\xi} \phi=\phi H, \quad \phi H+H \phi=0, H \xi=0, \quad \nabla_{\xi} \xi=0 .
\end{aligned}
$$

Proof. By (2.4), $\left(\nabla_{\xi} \phi\right) \xi=\phi \nabla_{\xi} \xi=0$, hence $\nabla_{\xi} \xi=0$, and $\nabla_{\xi} \eta=0$. Now, $g(\phi X, \phi Y)=$ $g(X, Y)-\eta(X) \eta(Y)$, yields

$$
\begin{aligned}
& 0=g\left(\left(\nabla_{\xi} \phi\right) X, \phi Y\right)+g\left(\left(\nabla_{\xi} \phi\right) Y, \phi X\right)=-g\left(\left(\nabla_{X} \phi\right) \xi, \phi Y\right)-g\left(\left(\nabla_{Y} \phi\right) \xi, \phi X\right) \\
& -2 g(\phi X, \phi Y)=g\left(\nabla_{X} \xi, Y\right)+g\left(\nabla_{Y} \xi, X\right)-2 g(\phi X, \phi Y) .
\end{aligned}
$$

With help of definition of $H, \nabla_{X} \xi=-\phi^{2} X+H X$. By $\phi \xi=0$, and $\eta(\phi X)=0$

$$
\begin{aligned}
& 0=\left(\nabla_{X} \phi\right) \xi+\phi \nabla_{X} \xi=-\left(\nabla_{\xi} \phi\right) X+\phi H X, \\
& 0=\eta\left(\left(\nabla_{X} \phi\right) Y\right)+\eta\left(\left(\nabla_{Y} \phi\right) X\right)=-g\left(\left(\nabla_{X} \phi\right) \xi, Y\right)-g\left(\left(\nabla_{Y} \phi\right) \xi, X\right) \\
= & g\left(\left(\nabla_{\xi} \phi\right) X, Y\right)+g\left(\left(\nabla_{\xi} \phi\right) Y, X\right)=g(\phi H X, Y)+g(\phi H Y, X) \\
= & g((\phi H+H \phi) X, Y) .
\end{aligned}
$$


Proposition 2. The fundamental form satisfies

$$
\begin{gathered}
3 d \Phi(X, Y, Z)=-3 g\left(\left(\nabla_{X} \phi\right) Y, Z\right)-\eta(Y) g(\phi X, Z)+\eta(Z) g(\phi X, Y) \\
-2 \eta(X) g(\phi Y, Z) . \\
d \Phi(X, Y, Z)-\eta(Z)\left(\nabla_{\xi} \Phi\right)(X, Y)=\frac{1}{4} g([\phi, \phi](X, Y), \phi Z)+2(\eta \wedge \Phi)(X, Y, Z) .
\end{gathered}
$$

Proof. From identities

$$
\begin{aligned}
3 d \Phi(X, Y, Z) & =\left(\nabla_{X} \Phi\right)(Y, Z)+\left(\nabla_{Y} \Phi\right)(Z, X)+\left(\nabla_{Z} \Phi\right)(X, Y), \\
{[\phi, \phi](X, Y) } & =-\phi\left(\nabla_{X} \phi\right) Y+\phi\left(\nabla_{Y} \phi\right) X+\left(\nabla_{\phi X} \phi\right) Y-\left(\nabla_{\phi Y} \phi\right) X .
\end{aligned}
$$

we obtain

$$
\begin{gathered}
3 d \Phi(X, Y, Z)=-g\left(\left(\nabla_{X} \phi\right) Y, Z\right)-g\left(\left(\nabla_{Z} \phi\right) X, Y\right)+g\left(\left(\nabla_{Y} \phi\right) X, Z\right) \\
=-3 g\left(\left(\nabla_{X} \phi\right) Y, Z\right)-2 \eta(X) g(\phi Y, Z)+\eta(Y) g(\phi Z, X)-\eta(Z) g(\phi Y, X), \\
\frac{1}{2}[\phi, \phi](X, Y)=-\phi\left(\nabla_{X} \phi\right) Y+\phi\left(\nabla_{Y} \phi\right) X+\eta(Y) X-\eta(X) Y .
\end{gathered}
$$

Hence

$$
\begin{gathered}
6 d \Phi(X, Y, Z)=-3 g\left(\left(\nabla_{X} \phi\right) Y-\left(\nabla_{Y} \phi\right) X, Z\right)+\eta(Y) g(\phi X, Z)-\eta(X) g(\phi Y, Z)+2 \eta(Z) g(\phi X, Y) \\
=\frac{3}{2} g([\phi, \phi](X, Y), \phi Z)+4 \eta(X) g(Y, \phi Z)+4 \eta(Y) g(Z, \phi X)+4 \eta(Z) g(X, \phi Y)+ \\
6 \eta(Z)\left(\nabla_{\xi} \Phi\right)(X, Y)=\frac{3}{2} g([\phi, \phi], \phi Z)+12(\eta \wedge \Phi)(X, Y, Z)+6 \eta(Z)\left(\nabla_{\xi} \Phi\right)(X, Y) .
\end{gathered}
$$

\section{Structure of nearly Kenmotsu Manifolds}

In this section, we will proof curvature relations for nearly Kenmotsu manifold. Let $R$ be Riemann curvature operator

$$
R(X, Y) Z=\left(\nabla_{X, Y}^{2} Z\right)-\left(\nabla_{Y, X}^{2} Z\right)=\left[\nabla_{X}, \nabla_{Y}\right] Z-\nabla_{[X, Y]} Z
$$

by the same letter we denote corresponding $(0,4)$-tensor

$$
R(X, Y, Z, W)=g(R(X, Y) Z, W) .
$$

Theorem 1. Let $(M, \phi, \xi, \eta, g)$ be nearly Kenmotsu manifold. We have following curvature relations

$$
\begin{gathered}
R(\phi X, Y, Z, W)+R(X, \phi Y, Z, W)+R(X, Y, \phi Z, W)+R(X, Y, Z, \phi W)=0, \\
R(\phi X, \phi Y, Z, W)=R(X, Y, \phi Z, \phi W),
\end{gathered}
$$

Proof. Let $T$ be $(1,3)$-tensor defined by (cf. (2.4)

$$
\left(\nabla_{X, Y}^{2} \phi\right) Z+\left(\nabla_{X, Z}^{2} \phi\right) Y=T(X, Y, Z),
$$

clearly $T(X, Y, Z)=T(X, Z, Y)$. For simplicity $T$ will also denote corresponding $(0,4)$ tensor

$$
T(X, Y, Z, W)=g(T(X, Y, Z), W) .
$$

From the Ricci identity,

$$
0=R(X, Y, Z, \phi W)-R(X, Y, W, \phi Z)-g\left(\left(\nabla_{X, Y}^{2} \phi\right) Z, W\right)+g\left(\left(\nabla_{Y, X}^{2} \phi\right) Z, W\right)
$$


eq. (3.4), and the first Bianchi identity, we find

$$
\begin{gathered}
R(X, Y, Z, \phi W)=R(X, Y, W, \phi Z)+g\left(\left(\nabla_{X, Y}^{2} \phi\right) Z, W-g\left(\left(\nabla_{Y, X}^{2} \phi\right) Z, W\right)\right. \\
=R(X, Y, W, \phi Z)-g\left(\left(\nabla_{X, Z}^{2} \phi\right) Y, W\right)+g\left(\left(\nabla_{Y, Z}^{2} \phi\right) X, W\right)+ \\
T(X, Z, Y, W)-T(Y, Z, X, W), \\
R(X, Y, Z, \phi W)=R(X, Z, Y, \phi W)-R(Y, Z, X, \phi W)=R(X, Z, Y, \phi W) \\
-R(Y, Z, W, \phi X)-g\left(\left(\nabla_{Y, Z}^{2} \phi\right)(X, W)+g\left(\left(\nabla_{Z, Y}^{2} \phi\right) X, W\right),\right.
\end{gathered}
$$

comparing right hand sides of these equations, we obtain

$$
\begin{gathered}
R(X, Z, Y, \phi W)-R(Y, Z, W, \phi X)-R(X, Y, W, \phi Z)+g\left(\left(\nabla_{Z, Y}^{2} \phi\right) X, W\right)+ \\
g\left(\left(\nabla_{X, Z}^{2} \phi\right) Y, W\right)+T(Y, Z, X, W)-T(X, Z, Y, W)=2 g\left(\left(\nabla_{Y, Z}^{2} \phi\right) X, W\right),
\end{gathered}
$$

we note, that

$$
\begin{gathered}
g\left(\left(\nabla_{Z, Y}^{2} \phi\right) X, W\right)+g\left(\left(\nabla_{X, Z}^{2} \phi\right) Y, W\right)=R(X, Z, Y, \phi W) \\
-R(X, Z, W, \phi Y)+T(Z, X, Y, W), \\
g\left(\left(\nabla_{Y, Z}^{2} \phi\right) X, W\right)=g\left(\left(\nabla_{Y, W}^{2} \phi\right) Z, X\right)-T(Y, W, Z, X),
\end{gathered}
$$

which being taken into account in (3.7), follow

$$
\begin{gathered}
2 R(X, Z, Y, \phi W)-R(X, Y, W, \phi Z)-R(Y, Z, W, \phi X)-R(X, Z, W, \phi Y)+ \\
T(Y, Z, X, W)+T(Z, X, Y, W)-T(X, Y, Z, W)+ \\
2 T(Y, W, Z, X)=2 g\left(\left(\nabla_{Y, W}^{2} \phi\right) Z, X\right) .
\end{gathered}
$$

By straightforward computations

$$
\begin{gathered}
T(Y, Z, X, W)+T(Z, X, Y, W)-T(X, Y, Z, W)+2 T(Y, W, Z, X)= \\
C(X, Y, Z, W)+2 g(\phi Y, W) g(H X, Z)+2 g(\phi X, Z) g(H Y, W)+ \\
2 g(\phi X, W) g(H Y, Z)+2 g(\phi Z, W) g(H X, Y)+ \\
2 g(\phi Z, X) g\left(Y, \phi^{2} W\right)+\eta(X) \eta(Y) g(\phi Z, W)-\eta(Z) \eta(Y) g(\phi X, W),
\end{gathered}
$$

where

$$
\begin{gathered}
C(X, Y, Z, W)=-\eta(Y) g\left(\left(\nabla_{Z} \phi\right) X, W\right)+\eta(Y) g\left(\left(\nabla_{X} \phi\right) Z, W\right) \\
-2 \eta(W) g\left(\left(\nabla_{Y} \phi\right) Z, X\right) .
\end{gathered}
$$

The anti-symmetrization of (3.10), in $Y$ and $W$, and the first Bianchi identity, follow

$$
\begin{gathered}
3 R(\phi X, Z, Y, W)+3 R(X, \phi Z, Y, W)+3 R(X, Z, \phi Y, W)+3 R(X, Z, Y, \phi W)+ \\
4 g(\phi Y, W) g(H X, Z)+4 g(\phi X, Z) g(H Y, W)+2 g(\phi X, W) g(H Y, Z) \\
-2 g(\phi X, Y) g(H W, Z)+2 g(\phi Z, W) g(H X, Y)-2 g(\phi Z, Y) g(H X, W)=0,
\end{gathered}
$$

now (3.1) will be proved, if $H=0$. We shall focus on the proof that $H=0$.

For $X=\xi(H \xi=\phi \xi=0)$ we obtain

$$
\begin{gathered}
R(\xi, \phi Z, Y, W)+R(\xi, Z, \phi Y, W)+R(\xi, Z, Y, \phi W)=0, \\
-R(\xi, Z, \phi Y, W)-R(\xi, \phi Z, Y, W)+\eta(Y) R(\xi, \phi Z, \xi, W)+ \\
R(\xi, \phi Z, \phi Y, \phi W)=0,
\end{gathered}
$$

hence

$$
R(\xi, Z, Y, \phi W)+R(\xi, \phi Z, \phi Y, \phi W)+\eta(Y) R(\xi, \phi Z, \xi, W)=0
$$


and

$$
\begin{gathered}
-R(\xi, Z, \phi Y, W)+R(\xi, \phi Z, Y, W)=\eta(W) R(\xi, Z, \xi, \phi Y) \\
-\eta(W) R(\xi, \phi Z, \xi, Y)+\eta(Y) R(\xi, \phi Z, \xi, W),
\end{gathered}
$$

by the last equation, we can simplify (3.13), to

$$
\begin{gathered}
3 R(\xi, \phi Z, Y, W)=2 \eta(Y) R(\xi, \phi Z, \xi, W)+2 \eta(W) R(\xi, Z, \xi, \phi Y) \\
-\eta(W) R(\xi, \phi Z, \xi, Y)-\eta(Y) R(\xi, Z, \xi, W) . \\
R(\xi, Z, \phi Y, \phi W)=0 .
\end{gathered}
$$

For $\nabla \xi=-\phi^{2}+H$,

$$
\begin{gathered}
\left.\left.R(Y, Z, \xi, X)=-g\left(\nabla_{Y} \phi^{2}\right) X, Z\right)+g\left(\nabla_{Z} \phi^{2}\right) X, Y\right) \\
-g\left(\left(\nabla_{Y} H\right) X, Z\right)+g\left(\left(\nabla_{Z} H\right) X, Y\right),
\end{gathered}
$$

taking cycling sum, by Bianchi identity

$$
g\left(\left(\nabla_{Z} H\right) X, Y\right)+g\left(\left(\nabla_{X} H\right) Y, Z\right)-g\left(\left(\nabla_{Y} H\right) X, Z\right)=0,
$$

hence

$$
\begin{gathered}
\left.R(Y, Z, \xi, X)=-g\left(\left(\nabla_{Y} \phi^{2}\right) X, Z\right)+g\left(\left(\nabla_{Z} \phi^{2}\right) X, Y\right)-g\left(\nabla_{X} H\right) Y, Z\right) \\
=\eta(Y) g(X, Z)-\eta(Z) g(X, Y)+\eta(Y) g(X, H Z)-\eta(Z) g(X, H Y) \\
-2 \eta(X) g(Z, H Y)-g\left(\left(\nabla_{X} H\right) Y, Z\right), \\
0=R(\xi, X, \phi Y, \phi Z)=-2 \eta(X) g(H \phi Y, \phi Z)-g\left(\left(\nabla_{X} H\right) \phi Y, \phi Z\right) \\
=2 \eta(X) g(H Y, Z)-g\left(\left(\nabla_{X} H\right) \phi Y, \phi Z\right) .
\end{gathered}
$$

Let take local unit eigenvector field $Y, \eta(Y)=0, H^{2} Y=\lambda Y$, note that $H^{2} \phi Y=-\lambda \phi Y$, as $\phi H+H \phi=0$, then

$$
\begin{gathered}
0=R(\xi, X, \phi Y, \phi H Y)=2 \lambda \eta(X)-g\left(\left(\nabla_{X} H\right) \phi Y, \phi H Y\right)=2 \lambda \eta(X) \\
-\frac{1}{2}\left(\left(\nabla_{X} H^{2}\right) \phi Y, \phi Y\right)=2 \lambda \eta(X)+\frac{1}{2} d \lambda(X),
\end{gathered}
$$

so $d \lambda=-4 \lambda \eta$, as $X$ is arbitrary, in consequence $\lambda=0$ or $d \eta=0$, and $H=0$.

To proof (3.2), let denote the left hand side of (3.1) by $\mathcal{R}_{l}$, then

$$
\begin{gathered}
0=\mathcal{R}_{l}(\phi X, Y, Z, W)+\mathcal{R}_{l}(X, \phi Y, Z, W)-\mathcal{R}_{l}(X, Y, \phi Z, W) \\
-\mathcal{R}_{l}(X, Y, Z, \phi W)=2 R(\phi X, \phi Y, Z, W)-2 R(X, Y, \phi Z, \phi W),
\end{gathered}
$$

now (3.3) is immediate.

Proposition 3. For nearly Kenmotsu manifold we have

$$
\left(\nabla_{\phi X} \phi\right) \phi Y+\left(\nabla_{X} \phi\right) Y-2 g(\phi X, Y) \xi+\eta(Y) \phi X=0 .
$$

Proof. By $\phi^{2}=-I d+\eta \otimes \xi$,

$$
\begin{gathered}
g\left(\left(\nabla_{X} \phi\right) \phi Y, Z\right)=g\left(\left(\nabla_{X} \phi\right) Y, \phi Z\right)+\eta(Z) g(X, Y)+ \\
\eta(Y) g(X, Z)-2 \eta(X) \eta(Y) \eta(Z),
\end{gathered}
$$

taking into account (2.4), we obtain

$$
\begin{gathered}
g\left(\left(\nabla_{\phi X} \phi\right) Y, Z\right)=g\left(\left(\nabla_{X} \phi\right) Y, \phi Z\right)+2 \eta(Y) g(X, Z) \\
-\eta(Z) g(X, Y)-\eta(X) \eta(Y) \eta(Z),
\end{gathered}
$$

the last above identities, together follow (3.24). 
Proposition 4. For nearly Kenmotsu manifold, we have the following relations

$$
\begin{gathered}
R(\xi, X, Y, Z)=\eta(Y) g(X, Z)-\eta(Z) g(X, Y), \\
\operatorname{Ric}(\phi Y, \phi Z)=\operatorname{Ric}(Y, Z)+2 n \eta(Y) \eta(Z), \\
\operatorname{Ric}(Z, \phi Y)+\operatorname{Ric}(\phi Z, Y)=0,
\end{gathered}
$$

where Ric indicates the Ricci tensor and $Q$ is the Ricci operator, $\operatorname{Ric}(X, Y)=g(Q X, Y)$.

Proof. Eq. (3.27) is direct consequence of $\nabla \xi=-\phi^{2}$, cf. (3.19).

Let $\left(E_{0}=\xi, E_{1}, \ldots, E_{n}, E_{n+1}, \ldots, E_{2 n}\right), \operatorname{dim} M=2 n+1$, denote orthonormal $\phi$-frame, $\phi E_{i}=E_{i+n}, \phi E_{i+n}=-E_{i}, i=1, \ldots, n$, then by (3.3),

$$
\begin{aligned}
& \operatorname{Ric}(X, Y)=\sum_{i=1}^{n}\left(R\left(E_{i}, X, Y, E_{i}\right)+R\left(E_{i+n}, X, Y, E_{i+n}\right)\right)+R(\xi, X, Y, \xi) \\
& =\operatorname{Ric}(\phi X, \phi Y)+\eta(X) \operatorname{Ric}(\xi, Y)-R(\xi, \phi X, \phi Y, \xi)+R(\xi, X, Y, \xi) \\
& =\operatorname{Ric}(\phi X, \phi Y)+\eta(X) R(\xi, Y)=\operatorname{Ric}(\phi X, \phi Y)+\operatorname{Ric}(\xi, \xi) \eta(X) \eta(Y),
\end{aligned}
$$

now by (3.27), $\operatorname{Ric}(\xi, \xi)=-2 n$, and the last identity is now direct consequence of (3.28).

Once we know that $d \eta=0$ we are able to describe completely local structure of nearly Kenmotsu manifold.

Theorem 2. Let $(M, \phi, \xi, \eta, g)$ be a nearly Kenmotsu manifold. Then

a) The distribution $D=\operatorname{ker} \eta$ is completely integrable, and maximall integral submanifolds of $D$ are totally umbilical hypersurfaces,

b) Maximall integral submanifolds naturally inherits nearly Kähler structure,

c) Nearly Kenmotsu manifold is locally isometric to warped product of real line and nearly Kähler manifold.

Proof. $\Rightarrow a)$ As Reeb form is closed, it is clear that $D=$ ker $\eta$ is completely integrable. If $\tilde{M}$, denote maximal integral submanifold of $D$, particularly $\operatorname{dim} \tilde{M}=2 n$, then restriction $\left.\xi\right|_{\tilde{M}}$ is normal vector field, and with respect to such choice of normal, Weingarten map is $A: \tilde{X} \mapsto \nabla_{\tilde{X}} \xi=-X$, hence $\bar{M}$ is umbilical.

$\Rightarrow b)$ Let $J$ be $(1,1)$ tensor field on $\tilde{M}$ defined by $J \tilde{X}=\phi \tilde{X}$. This definition is correct, as $D$ is $\phi$-invariant. It is direct that $J$ is almost complex structure. We verify

$$
\left(\tilde{\nabla}_{\tilde{X}} J\right) \tilde{X}=\tilde{\nabla}_{\tilde{X}} J \tilde{X}-J \tilde{\nabla}_{\tilde{X}} \tilde{X}=\left(\nabla_{\tilde{X}} \phi\right)(\tilde{X})=0,
$$

as $\eta(\tilde{X})=0$, and $\tilde{M}$ is totally umbilical.

$\Rightarrow c)$ We can choose coordinate neighborhood $U=I \times U^{\prime}$, where $I=(-\epsilon, \epsilon)$ is nonempty interval, and $U^{\prime} \subset \mathbb{R}^{2 n}$ is a disk, For any point $p \in U, p=\left(t, x^{1}, \ldots, x^{2 n}\right)=\left(t, p^{\prime}\right)$, coordinate $t$, can be defined in the way that $\eta=d t, \xi=\frac{\partial}{\partial t}$. If we set $\hat{g}=d t^{2}$ on $I$, then $\pi: p \mapsto t$ is Riemannian submersion, with fibers $\pi^{-1}(t)=t \times U^{\prime}$. We find that O'Neill tensors $A$, and $T^{0}$ vanish, hence $(U, g)$ is warped product $\left(I \times U^{\prime 2}+f^{2} h\right)$, as metric $h$ we may take $h=\iota_{0}^{*} g, \iota_{0}: p^{\prime} \mapsto 0 \times U^{\prime}$. As mean vector field $N=-2 n \xi$, we have $\pi_{*}(N)=-2 n \ln |f| \pi_{*}(\xi)$, hence $d \ln |f| / d t=1, f^{2}=C e^{2 t}$, and $C=1$, by our choice of $h$.

Remark 1. In 5], Dileo and Pastore proved special almost Kenmotsu manifold is locally isometric to warped product of real line and almost Kaehler manifold. 


\section{Some theorems about NeARly Kenmotsu manifolds}

In this section we will show that normal and nearly Kenmostu manifolds of constant sectional curvature are Kenmotsu. Moreover, nearly Kenmotsu manifold can be never realized as hypersurface of nearly Kähler manifold.

Theorem 3. Normal nearly Kenmotsu manifold is Kenmotsu.

Proof. We know that $d \eta=0$, hence nearly Kenmotsu manifold is normal iff $N=0$. But in view of the Proposition 2 in the case $N=0$, we have

$$
d \Phi=2 \eta \wedge \Phi
$$

which means that $M$ is almost Kenmotsu. Now we use the fact that normal almost Kenmotsu manifold is Kenmotsu.

The almost Hermitian manifold $(N, J, G)$ is called nearly Kähler if $\left(\bar{\nabla}_{X} J\right) Y+\left(\bar{\nabla}_{Y} J\right) X=$ 0, $\bar{\nabla}$ denotes the Levi-Civita connection of $G$, (see for more details [7], 9]). Simplest facts about nearly Kähler manifolds: If $N$ is Hermitian or locally flat then is Kähler, any four-dimensional nearly Kähler manifold is necessarily Kähler.

Y. Tashiro [15] proved that Riemannian hypersurface $(M, g) \subset(N, G)$, inherits almost contact metric structure $(\phi, \xi, \eta, g)$, where $(\phi, \xi, \eta)$ are defined by

$$
J X=\phi X+\eta(X) N, J N=-\xi,
$$

where $N$ is normal vector field.

Theorem 4. There is no nearly Kenmotsu hypersurface, in nearly Kähler manifold.

Proof. Let $A=-\bar{\nabla} N$, be Weingarten map, and $\nabla$ denote Levi-Civita connection on $M$. From $\left(\bar{\nabla}_{X} J\right) Y+\left(\bar{\nabla}_{Y} J\right) X=0$, Gauss-Weingarten equations follow

$$
\begin{gathered}
\left(\nabla_{X} \phi\right) Y+\left(\nabla_{Y} \phi\right) X-\eta(Y) A X-\eta(X) A Y+2 h(X, Y) \xi=0, \\
g\left(Y, \nabla_{X} \xi\right)+g\left(X, \nabla_{Y} \xi\right)=-h(Y, \phi X)-h(X, \phi Y),
\end{gathered}
$$

if $M$ is nearly Kenmotsu, then

$$
\begin{gathered}
-\eta(Y) \phi X-\eta(X) \phi Y=\eta(Y) A X+\eta(X) A Y-2 h(X, Y) \xi \\
h(X, Y)=h(\xi, \xi) \eta(X) \eta(Y) .
\end{gathered}
$$

In consequence $g\left(\nabla_{X} \xi, Y\right)+g\left(\nabla_{Y} \xi, X\right)=0$, which contradicts with Proposition 1.

Theorem 5. 9] Let $M$ be a nearly Kaehler manifold with $\operatorname{dim} M \leq 4$. Then $M$ is Kaehlerian.

Using Theorem 5 we can give following corollary.

Corollary 1. There is not exist proper nearly Kenmotsu manifolds for dimension 3 and 5 .

\section{REFERENCES}

[1] D. E. Blair, Riemannian geometry of contact and symplectic manifolds, Progress Math. Vol 203, Birkhäuser, Boston, MA, 2010.

[2] D. E. Blair, D. K. Showers and K. Yano, Nearly Sasakian structures, Kodai Math. Sem. Rep, 27, 175-180 (1976).

[3] J.-B. Butruille, Classification des variétés approximativement kähleriennes homogènes, Ann. Glob. Anal. Geom. 27, 201-225 (2005).

[4] B. Cappelletti Montano and G. Dileo, Nearly Sasakian Geometry and SU(2)-Structures, arXiv:1410.0942 2 . 
[5] G. Dileo and A. M. Pastore, Almost Kenmotsu manifolds and local symmetry, Bull. Belg. Math. Soc. 14, 343-354 (2007).

[6] H. Endo, On the curvature tensor of Nearly cosymplectic manifolds of constant $\Phi$-sectional curvature, An. Ştiint. Univ. Al. I. Cuza Iaşi. Mat., 51, no.2, 439-454 (2005).

[7] A. Gray, Nearly Kähler manifolds, J. Differential Geometry 4, 283-309 (1970).

[8] A. Gray, Riemannian manifolds with geodesic symmetries of order 3, J. Differential Geometry 7, 343-369 (1972).

[9] A. Gray, The structure of nearly Kähler manifolds, Math. Ann. 223, no.3, 233-248 (1976).

[10] K. Kenmotsu, A class of almost contact Riemannian manifold, Tohoko Math. J. 24, 93-103 (1972).

[11] Z. Olszak, Nearly Sasakian manifolds, Tensor, N. S., 33, 277-286 (1979).

[12] Z. Olszak, Five-dimensional Nearly Sasakian manifolds, Tensor, N. S., 34, 273-276 (1980).

[13] S. Sasaki, Lecture Notes on Almost Contact Manifolds, Part II. Tokohu University (1967).

[14] A. Shukla, Nearly trans-Sasakian manifolds, Kuwait J. Sci. Eng. 23, no.2, 139-144 (1996).

[15] Y. Tashiro, On contact structures of hypersurfaces in complex manifolds, Tohoku Math. J. 15, 50-62 (1963).

[16] K. Yano, M. Kon, Structures on Manifolds, World Scientific, Singapore (1984).

Uludag University, Faculty of Art and Science, Department of Mathematics, Gorukle 16059, BURSA-TURKEY

E-mail address: iremkupeli@uludag.edu.tr, piotrdacko@yahoo.com, cengiz@uludag.edu.tr 